\title{
Supernova VLBI
}

\section{Michael Bietenholz ${ }^{* \dagger}$}

Hartebeesthoek Radio Observatory, Krugersdorp, South Africa

E-mail: michael@hartrao.ac.za

VLBI is the only tool presently capable of resolving an extragalactic supernova in the first few years after the explosion. It thus allows us to study the expanding shell of ejecta and its interaction with its surroundings. I briefly review the results obtained so far, and report on new results from VLBI observations of supernovae including SN 1979C, SN 1986J, SN 1993J and SN 2008D. VLBI observations provide a direct constraint on the size and expansion velocity and deceleration of the supernova shock front. Monitoring the expansion of a supernova can provide information on the density profiles of both the circumstellar material and the ejecta, and thus on the mass-loss rate of the progenitor, as well as providing a geometric estimate of distances to the supernova with the expanding shock front method. So far, VLBI observations show that most supernovae seem to have shell structure, indicating interaction of the ejecta with the circumstellar medium. VLBI observations also provide constraints on relativistic ejecta, which are important in discussions concerning the relationship between SN I b/c and Gamma-ray bursters.

The 9th European VLBI Network Symposium on The role of VLBI in the Golden Age for Radio Astronomy and EVN Users Meeting

September 23-26, 2008

Bologna, Italy

* Speaker.

${ }^{\dagger}$ Also at York University, Toronto, Canada. 
Since the Galactic supernova rate is only on the order of one per century, with the last detected one occurring $\sim 300$ yr ago, we must look outside the Galaxy to study supernovae. At distances beyond the Magellanic Clouds, however, resolving a supernova in the first few decades of its life requires milliarcsecond resolution. This, in turn, requires very-long-baseline interferometry (VLBI), the only technology that allows sub-milliarcsecond resolution imaging.

The ejecta thrown out by a supernova explosion expand and interact with the circumstellar medium (CSM). This interaction gives rise to a forward shock driven into the CSM and a reverse shock driven back into the ejecta. These shocks, and associated instabilities, can both amplify the magnetic field and accelerate the particles, which are the ingredients necessary for synchrotron emission.

Only supernovae with a relatively dense CSM produce radio emission detectable with current technology, and so far, only core-collapse supernovae, that is, types Ib/c and II, have been detected in the radio. Almost all supernovae are first discovered optically, with only a fraction having detectable radio emission. If a supernova is radio-bright, then global VLBI at $\mathrm{cm}$ wavelengths can resolve the young supernova shell out to distances of $10-20 \mathrm{Mpc}$. So far, approximately 14 supernovae have been observed with VLBI (see table in reference [1]; for another recent review see [2]). Well resolved images, however, have been possible in only a few cases. With the exception of SN 1987A, which was resolved with the Australia Telescope Compact Array, only three supernovae have been sufficiently close and radio-bright to allow well-resolved VLBI imaging since shortly after the supernova explosion: SN 1986J (NGC 891, 10 Mpc) and SN 1993J (M81, 4 Mpc), and SN 1979C (M100, 16 Mpc), although several older supernovae in M82 also have well-resolved VLBI images [e.g., 3, 4].

The rewards of studying supernovae with VLBI are many. Resolving of the expanding shell of ejecta allows us to study in detail the interaction of the ejecta with the CSM, which is generally the stellar wind from the supernova progenitor. Over just a few years, such expanding supernova shocks allow us to probe the last $\sim 10,000$ yrs of wind history of the progenitor. VLBI imaging also allows us to study the evolution of the supernova shell, the shock acceleration process, and possibly the emergence of a black hole or a neutron star compact remnant. Finally VLBI studies of supernovae are also an important tool for measuring the supernova rate, and thus the star formation rate, in star-forming regions, which are typically highly obscured in the optical.

\section{SN 1993J}

SN 1993J remains the best studied radio supernova, with the possible exception of SN 1987A. It was amongst the brightest radio supernovae, reaching a peak flux density of $\sim 100 \mathrm{mJy}$. The first VLBI observations were obtained after only one month, and continue to the present, albeit with a reduced cadence. The VLBI images show a remarkably circular shell morphology $[5,6,7]$. The angular expansion velocity could be accurately measured, and it is the first supernova for which a changing deceleration rate could be measured [8, 9]. A direct distance to M81 of 3.96 $\pm 0.29 \mathrm{Mpc}$, slightly larger than the commonly used Cepheid distance, was determined using the "expanding shock front" method [10].

The expansion of the shell is very symmetrical about the explosion center [11,6]. In fact, astrometry shows that the expansion is circularly symmetrical about the explosion center to within 


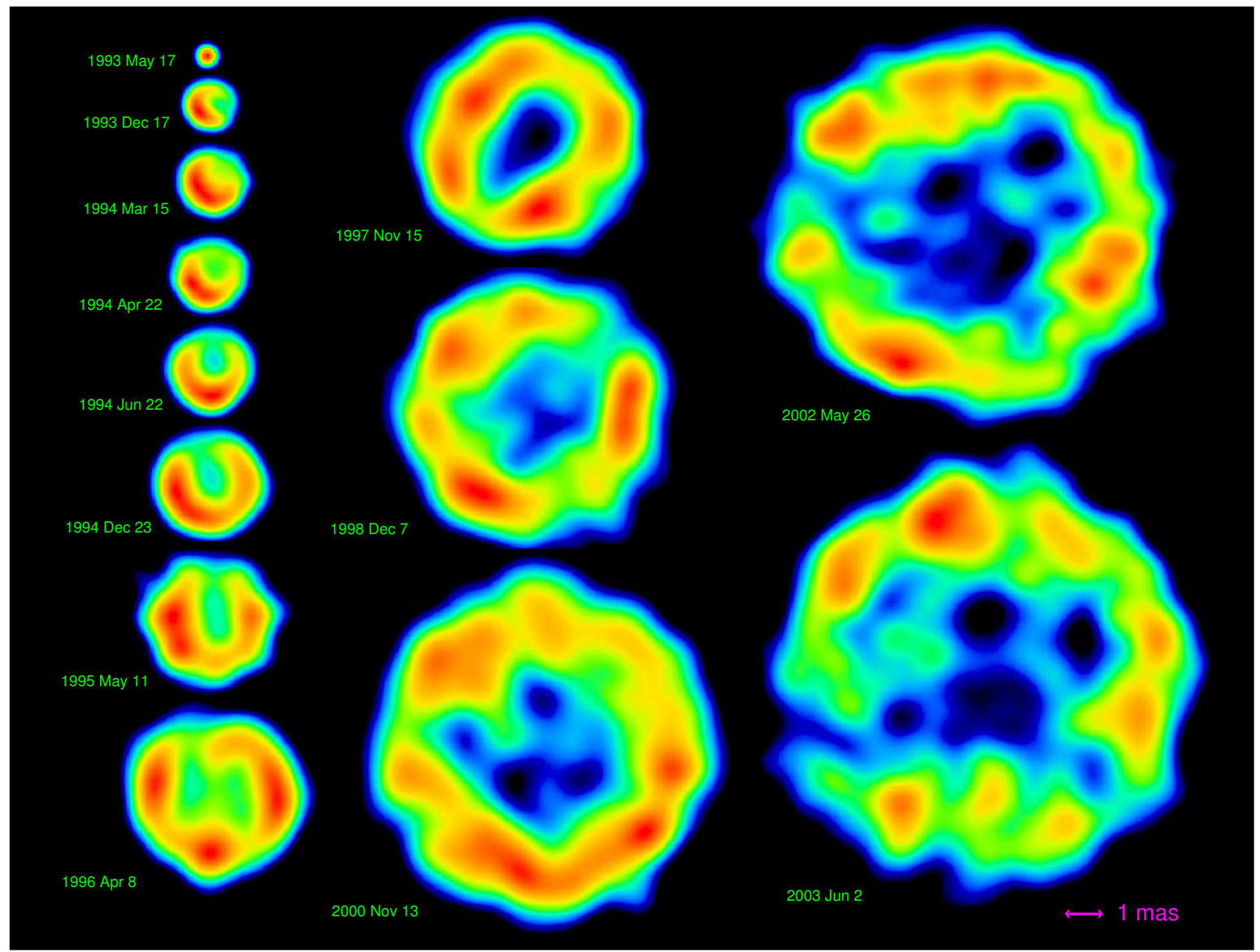

Figure 1: A sequence of VLBI images of SN 1993J from $50 \mathrm{~d}$ to $3171 \mathrm{~d}$ after shock breakout. The images in the first two columns are at $8.4 \mathrm{GHz}$ (see ref. [6]), while those in the rightmost column are at $5.0 \mathrm{GHz}$. The scale is shown at the lower right, north is up and east is to the left. The accompanying animation shows the expansion of the supernova, and was made from 27 epochs of VLBI observations (1993 to 2003) at 8.4 and $5 \mathrm{GHz}$.

$5.5 \%$ in projection. A sequence of images of SN 1993J, accompanied by an animation showing its expansion over a decade of VLBI imaging, is shown in Figure 1. At early times, there are clear, time-dependent deviations from circular symmetry, with brightness modulation by at least a factor of two [6]. Such modulation may persist to late times, however, the low signal-to-noise ratio makes it difficult to establish this conclusively. ${ }^{1}$

\section{SN 1986J}

Supernova 1986J is one of the very few supernovae discovered in the radio, probably about 3 years after the explosion. An early VLBI image of it marked the first time shell-like structure was

\footnotetext{
${ }^{1}$ It has been pointed out that some apparent deviations from circular symmetry can be due to deconvolution errors coupled to the $u-v$ plane sampling [12]. However, the systematic changes in the structure during the first five years or so cannot be due to such deconvolution errors because a) they are notably larger b) they are not bilaterally symmetric like deconvolution errors tend to be and, finally, c) the $u-v$ coverage of the observations does not evolve in any systematic way from epoch to epoch [see also discussion in ref. 6].
} 


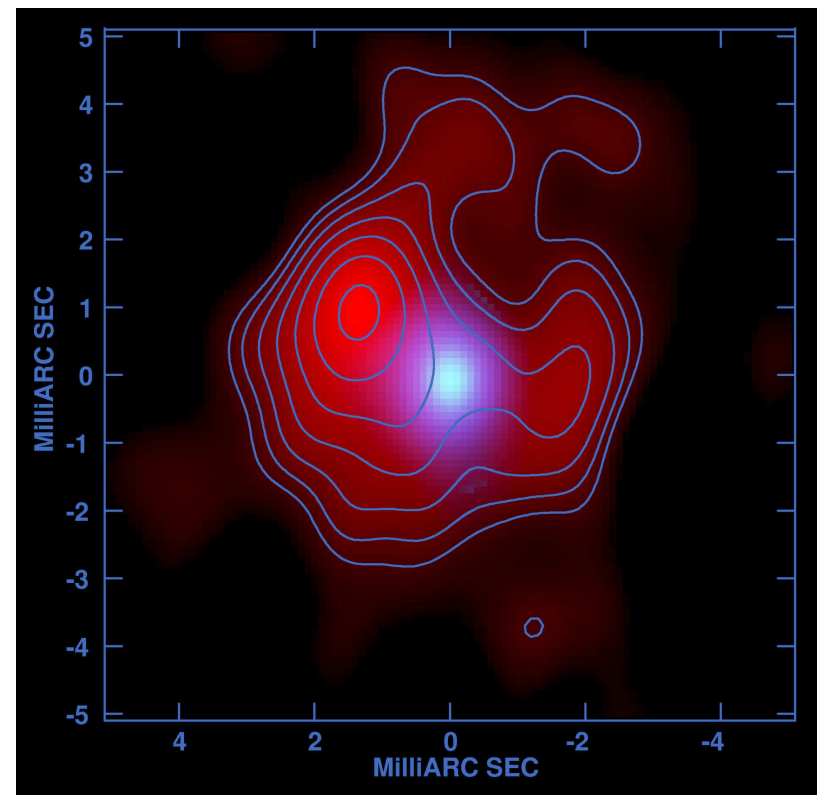

Figure 2: A dual-frequency VLBI image of SN 1986J, showing the compact, inverted-spectrum component located almost precisely in the center of the expanding shell. The red colour and the contours represent the $5 \mathrm{GHz}$ radio brightness, showing the shell emission. The contours are drawn at $11.3,16,22.6, \ldots, 90.5 \%$ of the peak $5 \mathrm{GHz}$ brightness of $0.55 \mathrm{mJy} \mathrm{bm}^{-1}$. The blue through white colors show the $15 \mathrm{GHz}$ radio brightness, showing the compact, central component which appeared around 1999. North is up and east to the left. The accompanying animation shows the expansion of the supernova, and the emergence of the central component.

seen in an IAU-designated supernova [13]. Its structure is rather more distorted than the text-book shell of SN 1993J, as can be seen in the image in Figure 2 (also accompanied by an animation showing the evolution of the supernova from almost 20 years of VLBI imaging). However, despite the distortions, the outline of the shell remains roughly circular, suggesting that the expansion is relatively symmetric, rather than, for example, being bipolar. SN 1986J also has the distinction of being the only supernova with a central component, which has a notably different radio spectrum than the shell, as can clearly be seen in the dual-frequency image in Figure 2. The nature of this component has not been conclusively established, however a possible candidate would be that it is emission associated with the compact remnant of the explosion, which in the case of SN 1986J could be either a black hole or a neutron star [14].

\section{SN 1979C}

This supernova, about $16 \mathrm{Mpc}$ away in M100 in the Virgo cluster, was one of the most optically and radio luminous Type II supernovae ever seen. The first VLBI observations were carried out in 1982 [15]. Since then, the expansion rate has been measured [16], leading to a substantially lower, and likely less model-dependent, estimate of the progenitor's mass-loss rate than does light-curve fitting [16]. The relatively large distance had prevented a determination of the morphology until 2005, when a new VLBI image showed SN 1979C to have a shell structure with an approximately 


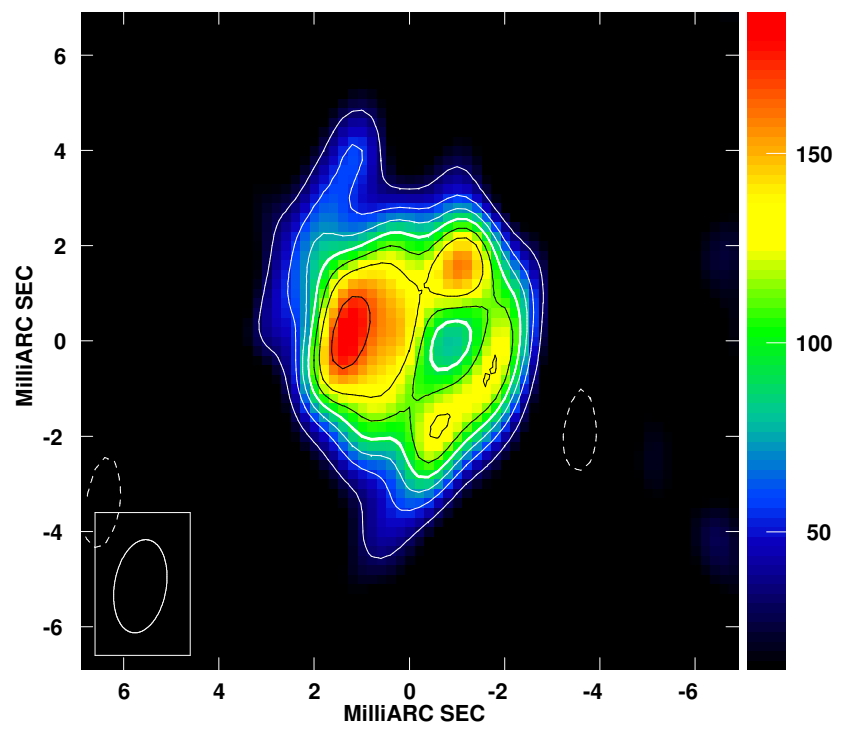

Figure 3: VLBI image of SN 1979C, at 5.0 GHz made using a global array on 2005 February 25. The contour levels are at $-17,17,30,40, \mathbf{5 0}$ (emphasized), 60, 70, and $90 \%$ of the peak brightness of $186 \mu \mathrm{Jy} \mathrm{bm}^{-1}$. The color scale, in $\mu \mathrm{Jy} \mathrm{bm}^{-1}$, is given at right, and the rms brightness of the background noise is 11 $\mu \mathrm{Jy} \mathrm{bm}^{-1}$. The FWHM of the restoring beam is shown at lower left. North is up and east to the left.

circular outline, similar to that of the other SNe which have been resolved. The VLBI image from [17] is shin Figure 3.

\section{Other Supernovae observed with VLBI}

A total of about a dozen more SNe have been observed with VLBI, including SN 1987A [18], SN 1996cr [19], SN 2001em (more below), SN 2001gd [20], SN 2004et [21], and SN 2007gr [22]. SN 1987A is close enough that its structure could be resolved both in radio optical and in X-rays [e.g., 23]. It has a shell structure at all three wavelengths.

In addition to studies of individual supernovae, wide-field VLBI images of star-forming galaxies have yielded fruitful results. Wide-field VLBI observations of the nearby starburst M82 have shown a number of supernovae and supernova remnants [e.g., 4]. The extreme starburst galaxy Arp 220 has been imaged with VLBI for over a decade, and the rate of new radio supernovae is $4 \pm 2 \mathrm{yr}^{-1}$ [24]. Radio observations of the starburst galaxy Arp 299 show that the rate of radio supernovae must be about 1 per year [25].

\section{SNe and GRBs: Relativistic Ejecta?}

It has been shown that long-duration gamma-ray bursts (GRBs) are associated with Type I b/c core-collapse supernovae. Superluminal expansion was in fact seen with VLBI in the case of GRB 030329/SN 2003dh [26]. As GRBs are thought to be highly beamed, off-axis GRB events should be at least $10 \times$ more common than the ones seen as GRBs. The prospect of resolving the jet of an off-axis GRB event associated with a Type I b/c supernova is therefore a tantalizing possibility. 
So far, however, this prospect continues to remain elusive: no emission clearly associated with an off-axis burst has yet been identified, although fairly strong afterglow is emission expected from such jets, particularly in the radio. For two relatively recent SNe, namely SN 2001em and SN 2008D, both of which were moderately bright in the radio, the presence of relativistic ejecta was suggested, based on their strong X-ray and/or radio emission. VLBI observations of both were carried out to provide direct observational constraints on the presence of relativistic ejecta. In both cases, the VLBI images showed no evidence for any radio-bright relativistic ejecta (for SN 2008D, see [27]; for SN 2001em, see [28] and [29] for the most recent results. Nonetheless, VLBI imaging of future candidates will provide the most definitive test of the presence and morphology of relativistic ejecta.

\section{Summary}

VLBI imaging has provided us with a great deal of information on young supernovae. The determination of the expansion speed and deceleration provide important constraints on the massloss history of the star, which are less model dependent than those available from light-curve fitting. As far as structure, all the supernovae that have been imaged with sufficient resolution so far show an edge brightened structure which is roughly circular in outline. ${ }^{2}$ This is the expected morphology of an optically thin spherical shell. This suggests that the maximum velocity of the supernova ejecta is isotropic, in other words the explosions tend not to be one-sided or bipolar in this respect. However, all the resolved supernovae also show significant asymmetries in the brightness, with onesided and bipolar and possibly more complex brightness enhancements of the basic shell structure being seen. This implies that significant density anisotropies, either in the CSM or in the ejecta, are a common feature. Since supernovae observable with VLBI are not common, it will be important to continue to exploit these opportunities to the fullest in the future.

\section{References}

[1] N. Bartel, Supernova VLBI, ArXiv e-prints 807 (July, 2008) [0807.0791].

[2] M. F. Bietenholz, Supernova VLBI in the present and with the SKA, ArXiv e-prints 802 (Feb., 2008) [0802.4219].

[3] D. M. Fenech, T. W. B. Muxlow, R. J. Beswick, A. Pedlar and M. K. Argo, Deep MERLIN $5 \mathrm{GHz}$ Radio Imaging of Supernova Remnants in the M82 Starburst, ArXiv e-prints (Oct., 2008) $[0810.0424]$.

[4] R. I. Beswick, D. Fenech, A. Pedlar and T. Muxlow, Long Term Monitoring of the SNR in M82, in The 9th European VLBI Network Symposium on The role of VLBI in the Golden Age for Radio Astronomy, Proceedings of Science, p. POS (IX EVN Symposium) 065, 2009.

\footnotetext{
${ }^{2} \mathrm{~A}$ possible exception is [MPW94] 41.95+57.5 in M82, althouth it is not clear that this object is in fact a supernova/supernova remnant [30].
} 
[5] N. Bartel, M. F. Bietenholz, M. P. Rupen, A. J. Beasley, D. A. Graham, V. I. Altunin, T. Venturi, G. Umana, W. H. Cannon and J. E. Conway, The Changing Morphology and Increasing Deceleration of Supernova 1993J in M81, Science 287 (Jan., 2000) 112-116.

[6] M. F. Bietenholz, N. Bartel and M. P. Rupen, SN 1993J VLBI. III. The Evolution of the Radio Shell, ApJ 597 (Nov., 2003) 374-398 [arXiv : astro-ph/0307382].

[7] J. M. Marcaide, A. Alberdi, E. Ros, P. Diamond, I. I. Shapiro, J. C. Guirado, D. L. Jones, F. Mantovani, M. A. Perez-Torres, R. A. Preston, R. T. Schilizzi, R. A. Sramek, C. Trigilio, S. D. van Dyk, K. W. Weiler and A. R. Whitney, Deceleration in the Expansion of SN 1993J, ApJL 486 (Sept., 1997) L31.

[8] N. Bartel, M. F. Bietenholz, M. P. Rupen, A. J. Beasley, D. A. Graham, V. I. Altunin, T. Venturi, G. Umana, W. H. Cannon and J. E. Conway, SN 1993J VLBI. II. Related Changes of the Deceleration, Flux Density Decay, and Spectrum, ApJ 581 (Dec., 2002) 404-426.

[9] J. M. Marcaide, A. Alberdi, M. A. Pérez-Torres, J. C. Guirado, L. Lara, E. Ros, P. J. Diamond, F. Mantovani, I. I. Shapiro, K. W. Weiler, R. A. Preston, R. T. Schilizzi, R. A. Sramek, C. Trigilio, S. D. van Dyk and A. R. Whitney, How is really decelerating the expansion of SN1993J?, in Proceedings of the 6th EVN Symposium, p. 239, June, 2002.

[10] N. Bartel, M. F. Bietenholz, M. P. Rupen and V. V. Dwarkadas, SN 1993J VLBI. IV. A Geometric Distance to M81 with the Expanding Shock Front Method, ApJ 668 (Oct., 2007) 924-940 [arXiv:0707.0881].

[11] M. F. Bietenholz, N. Bartel and M. P. Rupen, SN 1993J VLBI. I. The Center of the Explosion and a Limit on Anisotropic Expansion, ApJ 557 (Aug., 2001) 770-781 [arXiv:astro-ph/0104156].

[12] I. Heywood, K. M. Blundell, H.-R. Klöckner and A. J. Beasley, The radio remnant of SN1993J: an instrumental explanation for the evolving complex structure, MNRAS 392 (Jan., 2009) 855-867 [0810.5025].

[13] N. Bartel, M. P. Rupen, I. I. Shapiro, R. A. Preston and A. Rius, A high-resolution radio image of a young supernova, Nat 350 (Mar., 1991) 212-214.

[14] M. F. Bietenholz, N. Bartel and M. P. Rupen, Discovery of a Compact Radio Component in the Center of Supernova 1986J, Science 304 (June, 2004) 1947-1949.

[15] N. Bartel, A. E. E. Rogers, I. I. Shapiro, M. V. Gorenstein, C. R. Gwinn, J. M. Marcaide and $\mathrm{K}$. W. Weiler, Hubble's constant determined using very-long baseline interferometry of a supernova, Nat 318 (Nov., 1985) 25-30.

[16] N. Bartel and M. F. Bietenholz, SN 1979C VLBI: 22 Years of Almost Free Expansion, ApJ 591 (July, 2003) 301-315.

[17] N. Bartel and M. F. Bietenholz, Shell Revealed in SN 1979C, ApJ 682 (Aug., 2008) 1065-1069 [arXiv:0806.3482]. 
[18] D. L. Jauncey, A. Kemball, N. Bartel, I. I. Shapiro, A. R. Whitney, A. E. E. Rogers, R. A. Preston and T. A. Clark, Supernova 1987A - A radiosphere resolved with VLBI five days after the neutrino burst, Nat 334 (Aug., 1988) 412-415.

[19] F. E. Bauer, V. V. Dwarkadas, W. N. Brandt, S. Immler, S. Smartt, N. Bartel and M. F. Bietenholz, Supernova 1996cr: SN 1987A's Wild Cousin?, ApJ 688 (Dec., 2008) 1210-1234 [0804.3597].

[20] M. A. Pérez-Torres, A. Alberdi, J. M. Marcaide, M. A. Guerrero, P. Lundqvist, I. I. Shapiro, E. Ros, L. Lara, J. C. Guirado, K. W. Weiler and C. J. Stockdale, High-resolution observations of SN 2001gd in NGC 5033, MNRAS 360 (July, 2005) 1055-1062 [arXiv:astro-ph/0504647].

[21] I. Martí-Vidal, J. M. Marcaide, A. Alberdi, J. C. Guirado, L. Lara, M. A. Pérez-Torres, E. Ros, M. K. Argo, R. J. Beswick, T. W. B. Muxlow, A. Pedlar, I. I. Shapiro, C. J. Stockdale, R. A. Sramek, K. W. Weiler and J. Vinko, $8.4 \mathrm{GHz}$ VLBI observations of SN 2004et in NGC 6946, Astron. Astrophys. 470 (Aug., 2007) 1071-1077 [arXiv:0705.3853].

[22] Z. Paragi, C. Kouveliotou, M. A. Garrett, E. Ramirez-Ruiz, H. J. van Langevelde, A. Szomoru and M. Argo, e-VLBI detection of SN2007gr, The Astronomer's Telegram 1215 (Sept., 2007) 1.

[23] B. M. Gaensler, L. Staveley-Smith, R. N. Manchester, M. J. Kesteven, L. Ball and A. K. Tzioumis, Fifteen Years of High-Resolution Radio Imaging of Supernova 1987A, in American Institute of Physics Conference Series (S. Immler and R. McCray, eds.), vol. 937 of American Institute of Physics Conference Series, pp. 86-95, Oct., 2007. arXiv:astro-ph/0705.0057.

[24] R. Parra, J. E. Conway, P. J. Diamond, H. Thrall, C. J. Lonsdale, C. J. Lonsdale and H. E. Smith, The Radio Spectra of the Compact Sources in Arp 220: A Mixed Population of Supernovae and Supernova Remnants, ApJ 659 (Apr., 2007) 314-330 [arXiv:astro-ph/0612248].

[25] S. G. Neff, J. S. Ulvestad and S. H. Teng, A Supernova Factory in the Merger System Arp 299, ApJ 611 (Aug., 2004) 186-199 [astro-ph / 0406421 ].

[26] Y. M. Pihlström, G. B. Taylor, J. Granot and S. Doeleman, Stirring the Embers: High-Sensitivity VLBI Observations of GRB 030329, ApJ 664 (July, 2007) 411-415 [arXiv:0704.2085].

[27] M. F. Bietenholz, A. M. Soderberg and N. Bartel, Very-Long-Baseline Interferometry Observations of SN 2008D, ApJL 694 (Mar., 2009) L6-L10.

[28] M. F. Bietenholz and N. Bartel, SN 2001em: No Jet-driven Gamma-Ray Burst Event, ApJL 665 (Aug., 2007) L47-L50 [arXiv: 0706.3344 ]. 
[29] F. K. Schinzel, G. B. Taylor, C. J. Stockdale, J. Granot and E. Ramirez-Ruiz, SN 2001em: Not So Fast, ApJ 691 (Feb., 2009) 1380-1386 [0810 . 1478].

[30] T. W. B. Muxlow, A. Pedlar, R. J. Beswick, M. K. Argo, T. J. O'Brien, D. Fenech and W. Trotman, Is 41.95+575 in M82 actually an SNR?, Memorie della Societa Astronomica Italiana 76 (2005) 586. 